\title{
Antidementic activity of Brassica oleracea I. var. Italica (brassicaceae) flower on memory deficit in young male rats
}

\begin{abstract}
Memory deficit is major health problem in normal life and in numerous psychiatric and neurodegenerative states during which patients forget recent happenings and become profoundly disabled, mute, immobile over 5-10years. Brassica oleracea L. var. Italica (Broccoli) was investigated for its anti-dementic activity on memory deficit induced by scopolamine $(0.3 \mathrm{mg} / \mathrm{kg}$, i.p. $)$ in young male rats using behavioural models [elevated plus maze (EPM) \& Morris water maze (MWM)]. Brain malondialdehyde (MDA), nitrite and reduced glutathione (GSH) biomarkers were also estimated to assess oxidative potential of broccoli. Its flower extract was prepared with hydro-alcohol (1:1) solvent using Soxhlet apparatus. Two doses $(40 \mathrm{and} 80 \mathrm{mg} / \mathrm{kg}$, p. o.) of Hydro-alcoholic flower extract of Brassica oleracea L. var. Italica (HFEBOI) were administered for four consecutive days to separate groups of rats. Chronic pretreatment with HFEBOI (40 and $80 \mathrm{mg} / \mathrm{kg}$, p. o.) significantly and dose dependently improved learning and memory of rats, as indicated by increase in inflation ratio using EPM, and decrease in ELT during acquisition and increase in time spent in target quadrant (TSTQ) during retrieval using MWM. The drugs did not show any significant effect on loco-motor activity of the rat. Oxidative stress biomarkers (elevated brain MDA, nitrite and lower GSH levels) in scopolamine induced memory deficit in rats were significantly reversed by the flower extract. HFEBOI significantly reversed scopolamine induced memory deficit in rats. HFEBOI also significantly reduced brain acetylcholinesterase (AChE) activity of rats. The results indicated potential of HFEBOI in relieving memory deficit and oxidative stress induced by scopolamine. Anti-dementic mechanism of Brassica oleracea L. var. Italica may be mediated through the inhibition of brain AChE activity by diindolylmethane, an anti-ChE and oxidative stress leading to rejuvenation of central nervous system that relieves dementia.
\end{abstract}

Keywords: memory, Brassica oleracea 1, scopolamine; memory deficit, broccoli, scopolamine, anticholinesterase, elevated plus maze, morris water maze, inflation ratio, HFEBOI
Volume 5 Issue 3 - 2017

\author{
Anuradha Madhukar,Vrish Dhwaj Ashwlayan \\ Department of Pharmaceutical Technology, M.I.E.T. Meerut, Dr. \\ A.P.J.Abdul Kalam Technical University, India
}

Correspondence: Vrish Dhwaj Ashwlayan, Department of Pharmaceutical Technology, Meerut Institute of Engineering and Technology (M.I.E.T.), India, Tel 9194I2493228, Fax 91 1212439058,Email vrishsps@gmail.com

Received: January 29, 2017 | Published: May 30, 2017
Abbreviations: AChE, acetylcholinesterase; Anti-ChE, anticholinesterase; BP, boiling point; DTNB, 5, 5'-dithio-bis 2nitrobenzoic Acid; ELT, escape latency time; EPM, elevated plus maze; GSH, reduced glutathione; HFEBOI, hydro-alcoholic flower extract of Brassica oleracea L. italic; IR, inflation ratio; MDA, malondialdehyde; MWM, morris water maze; NO, nitric oxide; ROS, reactive oxygen species; TBA, thiobarbituric acid; TCA, trichloroacetic acid; TLT, transfer latency time; TSTQ, time spent in target quadrant

\section{Introduction}

Dementia is a several pathological states of brain (Alzheimer's, Parkinson's, prion and Huntington's disease and spinal muscular atrophy) leading to disruption of multiple higher cortical functions including memory, reasoning, orientation, comprehension, learning capacity and emotional stability. ${ }^{1}$ Vascular dementia with cerebral disease is now the third most common cause of dementia. ${ }^{2}$ Senile dementia (memory loss and disorientation in Alzheimer's disease) is progressive neurodegenerative condition (neuronal injury, synaptic failure and cortical cholinergic neuronal death) in elders characterized by deposition of senile plaques of amyloid beta $(A \beta)$ in neocortex and formation of neurofibrallry tangles in hippocampus. ${ }^{3}$ Synaptic loss due to decrease in synapthophysin is greater in pre-senile (60-65years) onset than in senile (90-95years) onset. ${ }^{4}$ It is the common and fatal form of dementia possibly contributes to $60-70 \%$ of cases. Cognitive deficits are major health problem in normal life and in numerous psychiatric and neurodegenerative states ${ }^{5}$ during which patients forget recent happenings and become profoundly disabled, mute, immobile over 5-10years. ${ }^{6}$ Its physical symptoms are development of involuntary movement in the fingers, feet, face or trunk, increased clumsiness, loss of coordination and balance, slurred speech, jaw clenching or teeth grinding, difficulty in swallowing or eating, continual muscular contractions, stumbling or falling. Central cholinergic neurons in the basal forebrain innervate the cortex and hippocampus. Hypo-function of cholinergic neurons may induce senile dementia. AChE plays a key role in the regulation of the cholinergic system and hence, drugs that inhibits AChE enzyme which breaks down acetylcholine are known to prevent senile dementia. Nootropic represents psycotropic agents such as piracetam and cholinesterase inhibitors like donepezil with selective facilitatory effect on intellectual performance, learning capability and memory in both senile and vascular dementia especially in the early and moderate stages. ${ }^{7}$ The miosis, salivation, hypothermia and tremor side effects ${ }^{8}$ have made their applicability limited. ${ }^{9}$ Medicinal plants attracted attention due to their potential role in dementia. ${ }^{10}$ Broccoli, 
a crown of vegetables belongs to plant Brassica oleracea L. Italica Plenk of Brassicaceae family has large flower heads, usually green in colour arranged in a tree like structure branching out from a thick and edible stalk with unpleasant smell and bitter taste. Broccoli is an Italian word from the Latin brachium, meaning an arm or branch. The word 'Brassica' means to cut off the head. Its leaf, seeds and flowers are the parts used for anti-oxidative supplement, detoxification activity and beauty function in the indigenous medicine. ${ }^{11} \mathrm{LD}_{50}$ test was performed on broccoli florets crude extract, no toxicity was shown in the crude broccoli extract up to $10 \mathrm{~g} / \mathrm{kg}$ body weight in rats. ${ }^{12}$ The flower possesses as gastroprotective, antimicrobial,${ }^{13}$ antioxidant, anticancer, hepatoprotective, ${ }^{14}$ cardioprotective, anti-obesity, antidiabetic, anti-inflammatory, immunomodulatory ${ }^{15}$ and anticonvulsant activities. ${ }^{16}$ Broccoli is nutritional powerhouse of health promoting compounds such as diindolylmethane, vitamin A, C, E, poly-phenols, suloraphane, glucosinolates antioxidants, hydroxycinnamic acids and omega-3 fatty acid. ${ }^{17}$ Suloraphane, an isothiocynate sulfur containing component of broccoli is long lasting antioxidant and detoxifier, has been shown to stop over-rapid ageing by restoring antioxidant gene expression in human epithelial and promoting body's immune defense system. Herbal compounds are known to encompass higher antioxidant potential as compared to synthetic compound. Diindolylmethane of broccoli possessed anti-cholinesterase (anti-ChE) activity. Therefore, aim of the present research work is to investigate antidementic activity of Brassica oleracea L. var. Italica using interceptive (scopolamine induced memory deficit) and exteroceptive (EPM and MWM) behavioural models in young male rats and to understand its mechanism in dementia.

\section{Material and methods}

Experimental Animals: Wistar male rats of 6weeks age $(150 \pm 2 \mathrm{~g})$ were procured from IVRI, Izzatnagar, Bareilly (Uttar Pradesh) India. They were housed in animal house provided with 12 hours light and dark cycles at $25 \pm 2{ }^{\circ} \mathrm{C}$ and had free access to water and standard laboratory diet. The experimental protocol was approved by the institutional animal ethics committee (no. 711/PO/Re/S/02/ CPCSEA) and experiments were conducted according to the CPCSEA guidelines on the use and care of experimental animals. Experiments were carried out between 09:00 and 17:00hours. Efforts were made to minimize animal suffering and number of animals used.

Drugs and chemicals: Piracetam, Scopolamine hydrobromide drugs were purchased from Sigma Aldrich. Petroleum ether, ethanol, chemicals were purchased from Fisher scientific. Analytical reagent grade chemicals were used in study.

Plant material: The flower of Brassica oleracea Linn was purchased from market. The flower was authenticated by Dr. R. S. Saxena Reader and Head, Botany Department, Meerut College, Meerut. A voucher specimen was deposited for the plant in the same herbarium.

Extraction: Air dried flower (20g) of Brassica oleracea Linn (Brassicaceae) was coarsely powdered and extracted with petroleum ether (B. P. $40-60^{\circ} \mathrm{C}$ ) for defatting of crude drug followed by water and ethanol (hydro-alcohol 1:1) HPLC grade solvents (B. P. $78.37^{\circ} \mathrm{C}$ ) for 18 hours, under reflux by continuous hot percolation method using "Soxhlet apparatus". ${ }^{18}$ This was repeated thrice with fresh solvent each time. The extracts from all the three washes were pooled and concentrated using Rota evaporator (Perfit) to obtain dark viscous mass. The per cent yield of HFEBOI was found to be $6.38 \%$. The extract was suspended in normal saline $(0.9 \% \mathrm{w} / \mathrm{v}$ sodium chloride $)$ solution. The residue was then dried at room temperature. The extract was subjected to phytochemical analysis as per protocols. ${ }^{19}$ Phytochemical screening of the HFEBOI flower extract indicated presence of alkaloids, carbohydrates, glycosides, phenolic compounds, flavonoids, amino acids and proteins.

Intrceptive behavioural model (Scopolamine- induced Memory deficit): Memory deficit in rats is induced by scopolamine $(0.3 \mathrm{mg} / \mathrm{kg}$, i.p.), by blocking postsynaptic muscarinic cholinergic receptors. ${ }^{20}$

\section{Extroceptive behavioural models}

\section{EPM}

EPM served as the exteroceptive behavioural model to evaluate learning and memory in rats. The apparatus consists of two open arms $(50 \mathrm{~cm} \times 10 \mathrm{~cm})$ and two covered arms $(50 \mathrm{~cm} \times 10 \mathrm{~cm} \times 40 \mathrm{~cm})$. The arms extend from a central platform $(10 \mathrm{~cm} \times 10 \mathrm{~cm})$, and maze is elevated to a height of $50 \mathrm{~cm}$ from the floor. Acquisition of memory was recorded on day 4 after scopolamine administration. On the first day, each rat is placed at the end of an open arm, facing away from the central platform. Time taken by the rat to move into any one of the covered arms with all its four legs is recorded as TLT on the first day (i.e., $4^{\text {th }}$ day of drug administration) for each animal. If the rats do not enter into one of the covered arms within 90seconds, they are gently pushed into one of the two covered arms and the TLT is assigned as 90 seconds. The rat was allowed to explore the maze for $10 \mathrm{sec}$ and then return to its home cage. Memory retention was calculated after $24 \mathrm{hrs}$ of acquisition trial (on the $5^{\text {th }}$ day) as inflation ratio using the following formula described by Inflation Ratio (IR) $=\mathrm{L}_{1}-\mathrm{L}_{0 /} \mathrm{L}_{0}$ where $\mathrm{L}_{0}$ is initial TLT on day 4 in seconds and $\mathrm{L}_{1}$ is the TLT after $24 \mathrm{hrs}$ (fifth day) of acquisition trial. ${ }^{21}$

\section{MWM}

MWM model is employed to evaluate learning and memory. ${ }^{22} \mathrm{It}$ consists of a circular water tank (diameter $150 \mathrm{~cm}$ and height $45 \mathrm{~cm}$ ), filled with water maintained at $25^{\circ} \mathrm{C}$. The water is made opaque with a white coloured dye. The tank is divided into four equal quadrants with the help of two threads, fixed at right angle to each other on the rim of the pool. A platform $(10 \times 10 \mathrm{~cm})$ of $29 \mathrm{~cm}$ height is located in the centre of one of these four quadrants. The position of platform and clues are kept consistent throughout the training session. In the present study, target quadrant is Q1.

\section{Acquisition trials}

Each rat was subjected to four consecutive trials on each day with an interval of five minutes, during which rat was allowed to escape on the hidden platform and was allowed to remain there for 20 seconds. In case of inability of the rat to locate the hidden platform within 90 seconds, it was gently guided by hand to the platform and allowed to remain there for 20seconds. Escape latency time (ELT) to locate the hidden platform in water maze is noted as index of acquisition and learning during acquisition trials. In preliminary study, trial was conducted to familiarize the rats with the task and was not counted. Rat was subjected to acquisition trials for four consecutive days. Extensive pretraining is not required in this model because animals learn rapidly to locate the hidden platform. The starting position on each day to conduct four acquisition trials was changed as given below:
Day 1
Q1
Q2
Q3
Q4
Day 2
Q2
Q3 


$\begin{array}{lllll}\text { Day 3 } & \text { Q3 } & \text { Q4 } & \text { Q1 } & \text { Q2 } \\ \text { Day 4 } & \text { Q4 } & \text { Q1 } & \text { Q2 } & \text { Q3 }\end{array}$

\section{Retrieval trial}

On the next day, platform was removed and each rat was allowed to explore the pool for 120 seconds. Mean time spent by the mouse in each of four quadrants was noted. The mean time spent by the rat in target quadrant (Q1) for searching the hidden platform was noted as an index of retrieval. The mean time spent in target quadrant (TSTQ) by the rat for searching the hidden platform was noted as an index of retrieval conducted on day 5 .

\section{Experimental protocol}

Rats were allocated into sixteen groups and each group contained six rats.

In group I (control), rats were administered $0.9 \% \mathrm{w} / \mathrm{v}$ sodium chloride solution $(10 \mathrm{ml} / \mathrm{kg}$, i.p.) on first day and again after 24hours i.e., on the second day. In group II and III, rats were administered scopolamine hydrobromide $(0.3 \mathrm{mg} / \mathrm{kg}$, i.p. $)$ and piracetam $(400 \mathrm{mg} /$ $\mathrm{kg}$ ) respectively. TLT was recorded after 45 minutes in groups I \& II; after $60 \mathrm{~min}$ in group III respectively and then after 24 hours (the second day) using EPM. In group IV and V, rats were administered HFEBOI at doses of 40 and $80 \mathrm{mg} / \mathrm{kg}$, p.o. respectively for four consecutive days. In group IV and V, TLT was recorded after 60minutes (the fourth day) and then after 24hours (the fifth day) using EPM. In groups VI, VII and VIII, rats were administered piracetam $(400 \mathrm{mg} / \mathrm{kg}$, i.p.) and scopolamine hydrobromide $(0.3 \mathrm{mg} / \mathrm{kg}$, i.p.); HFEBOI $(40$ and $80 \mathrm{mg} /$ $\mathrm{kg}$, p.o.) and scopolamine hydrobromide $(0.3 \mathrm{mg} / \mathrm{kg}$, i.p.) 60 and $45 \mathrm{~min}$ respectively, before the first day exposure on EPM. TLT was recorded on the fourth and then fifth day in VI to VIII groups. Group IX (control) of normal saline solution $(10 \mathrm{ml} / \mathrm{kg}$, i.p.) treated rats was subjected to MWM for measuring ELT (from day 1 to day 4) and TSTQ on the fifth day. In group X and XI, rats were administered scopolamine hydrobromide $(0.3 \mathrm{mg} / \mathrm{kg}$, i.p. $)$ and piracetam $(400 \mathrm{mg} /$ $\mathrm{kg}$, i.p.) respectively before 45 minutes of acquisition trial conducted on four consecutive days (from day 1 to day 4). In case of group XII and XIII, HFEBOI at doses of 40 and $80 \mathrm{mg} / \mathrm{kg}$, p.o., respectively were administered for four days before 60 minutes of acquisition trials conducted on four consecutive days (from day 1 to day 4). In groups XIV, XV and XVI rats were administered, HFEBOI $(40$ and $80 \mathrm{mg} / \mathrm{kg}$, p.o.) and scopolamine hydrobromide $(0.3 \mathrm{mg} / \mathrm{kg}$, i.p.); and piracetam $(400 \mathrm{mg} / \mathrm{kg}$, i.p.) and scopolamine hydrobromide $(0.3 \mathrm{mg} / \mathrm{kg}$, i.p.) 60 and 4 min respectively, before the acquisition trials conducted on four consecutive days (from day 1 to day 4 ). In all the above mentioned groups, $0.9 \% \mathrm{w} / \mathrm{v}$ sodium chloride solution $(10 \mathrm{ml} / \mathrm{kg}$, i.p.) was administered 45 minutes before retrieval trial conducted on the fifth day.

\section{Estimation of oxidative stress markers in brain homogenate}

On the fifth day immediately after behavioural testing (retrieval), rat was euthanized by overdose of $2.5 \%$ thiopental sodium $(25 \mathrm{mg} /$ $\mathrm{kg}$, i.p.). It produces rapid induction with minimum excitation. The whole brain was carefully removed from the skull. For preparation of brain homogenate, the fresh whole brain was weighed and transferred to a glass homogenizer and homogenized in an ice bath after adding 10 volumes of phosphate buffer $(\mathrm{pH} 8,0.1 \mathrm{M})$. The homogenate was centrifuged using refrigerated centrifuge at $3000 \mathrm{rpm}$ for $10 \mathrm{~min}$ at $4^{\circ} \mathrm{C}$. Drug induced lipid per-oxidation was measured by estimation of the content of MDA, nitrite, GSH, and AChE in brain blood sample of the rats. The determination was done by precipitating the protein substance using trichloroacetic acid $(10 \% \mathrm{w} / \mathrm{v})$, the protein free sample used for estimation of lipid per-oxidation parameters as follows:

\section{Protocol for the estimation of brain malondialdehyde (MDA)}

MDA was formed as an end product of lipid per-oxidation, which reacts with thiobarbituric acid and forms faint pink coloured trimethene complex. $1 \mathrm{ml}$ of supernatant was taken. $0.5 \mathrm{ml}$ of $30 \%$ of Trichloro acetic acid (TCA) and $0.3 \mathrm{ml}$ of $0.8 \%$ of thiobarbituric acid (TBA) were added in test tubes. The tubes were covered by the aluminum foil and then heated in water bath at $90^{\circ} \mathrm{C}$ for $15 \mathrm{~min}$. Then the mixture was kept in ice cool water for $30 \mathrm{~min}$. After cooling, the absorbance of the colour supernatant in $1 \mathrm{ml}$ butanol was measured at $532 \mathrm{~nm}$ using UV spectrophotometer. ${ }^{23}$ MDA level was expressed in $\mu \mathrm{M} / \mathrm{mg}$.

\section{Protocol for the estimation of brain Nitrite}

The accumulation of nitrite in the supernatant, an indicator of the production of nitric oxide, was determined by a colourimetric assay with the Griess reagent ( $0.1 \% \mathrm{~N}-(1$-napththyl) ethylenediamine dihydrochloride, $1 \%$ sulfanilamide and $5 \%$ phosphoric acid). Equal volumes of the supernatant and the Griess reagent were mixed and incubated for $10 \mathrm{~min}$ at room temperature in the dark. The absorbance was taken at $542 \mathrm{~nm}$ using a spectrophotometer. The concentration of nitrite in the supernatant was determined from a sodium nitrite standard curve. ${ }^{24}$

\section{Protocol for the estimation of brain reduced glutathione (GSH)}

GSH was estimated by colorimetric method. GSH level was expressed in $\mu \mathrm{M} / \mathrm{mg}$ of protein. The assay contained $1 \mathrm{ml}$ supernatant and $1 \mathrm{ml}$ of $10 \%$ of TCA. This mixture was centrifuged for $10 \mathrm{~min}$ at 3000-4000 gyrations. Again the supernatant was collected and $10 \mu 1$ of supernatant, $2 \mathrm{ml}$ of $0.1 \mathrm{M}$ phosphate buffer (pH-7.4), $0.5 \mathrm{ml}$ of DTNB $(5,5$ '-dithio-bis 2-nitrobenzoic acid) and $0.4 \mathrm{ml}$ of distilled water were added and mixed. Absorbance of this mixture was measured at $412 \mathrm{~nm}$ within 15 minutes. ${ }^{25}$

\section{Protocol for the estimation of brain AChE activity}

The resultant cloudy supernatant liquid was used for the estimation of brain AChE activity. The whole brain AChE activity was expressed in $\mu \mathrm{M} / \mathrm{min} / \mathrm{mg}$ of protein. The end point was the formation of the yellow colour because of the reaction of thiocholine with dithiobisnitrobenzoate (DTNB) ions. The rate of formation of thiocholine from acetylcholine iodide in the presence of tissue cholinesterase was measured using spectrophotometer. The resulting yellow colour was due to reduction of DTNB by certain substances in the brain homogenate and due to non-enzymatic hydrolysis of substrate. After having calibrated the instrument, the change in absorbance per min of sample was read at $412 \mathrm{~nm}$. The rate of hydrolysis of substrate was calculated using following formula:

$\mathrm{R}=$ change in absorbance $/ \mathrm{min} \times 5.74 \times 10^{-4} / \mathrm{C} 0$

Where, $\mathrm{R}$ = rate of hydrolysis of acetylcholine iodide $/ \mathrm{min} / \mathrm{mg}$ tissue, $\mathrm{C} 0=$ weight of tissue homogenate $\mathrm{inmg} / \mathrm{mL}$.

\section{Loco-motor activity measurement by rota-rod}

The ability of rat to hold on to horizontally rotating rod (diameter $2.5 \mathrm{~cm}, 4$ rotations per min) was used to assess motor coordination. 
Motor co-ordination was considered to be impaired if the rat felloff from the rotating-rod within ninety seconds. In control or drug treated groups, the assessment of motor coordination was made before and after administration of vehicle or drugs during acquisition and retrieval trials. There is no difference between treatment groups with regard to exclusion because animals that demonstrated ataxia with or without drug treatment were not included in study. $10 \%$ exclusion rate was noted. ${ }^{26}$

\section{Statistical analysis}

The values were expressed as mean \pm SEM for 6 animals. The results were subjected to statistical analysis by using one-way ANOVA in MWM and EPM models to calculate the significant difference, if any, among the groups $p \leq 0.05$ was considered as significant.

\section{Results and discussion}

\section{HFEBOI improved on behavioural alteration in scopolamine treated rats}

The HFEBOI was evaluated for antidementic activity in prevention of scopolamine induced memory deficit using EPM and MWM Models. Hydro-alcoholic extract of flower was administered for four consecutive days in rats at 40 and $80 \mathrm{mg} / \mathrm{kg}$, p. o. doses which had no acute behavioural effect.

\section{EPM}

HFEBOI was investigated for its effect on scopolamine induced memory deficit using EPM. Control, piracetam, HFEBOI per se treated rats entered closed arm quickly as compared to scopolamine treated rats. Mean retention TLT $\left(\mathrm{L}_{1}\right)$ to enter closed arm on day 5 was shorter as compared to initial TLT $\left(\mathrm{L}_{0}\right)$ on day 4 of each group indicating IR (Figure 1). In contrast, significant decrease in TLT as IR noted in scopolamine treated group as compared to normal saline treated control group indicating impairment of memory retention. Administration of HFEBOI (40 and $80 \mathrm{mg} / \mathrm{kg}$, p. o.) prior to scopolamine increased IR significantly $(\mathrm{p} \leq 0.05)$ as compared to scopolamine treated group indicating the reversal of scopolamine induced memory deficit (Figure 2).

\section{MWM}

Control, piracetam, HFEBOI (40 and $80 \mathrm{mg} / \mathrm{kg}$, p. o.) per se treated rats quickly learned to swim directly to the platform in the MWM on day 4 and produced significantly increase in TSTQ on day 5 as compared to control group indicating normal acquisition and retrieval of memory. Scopolamine treated rats showed significant increase in ELT, which declined with continued training during the acquisition of a spatial navigation task on day 4 (Table 1) and decrease in TSTQ markedly as compared to target quadrant (Q1) of control group indicating impairment in acquisition and retrieval components of memory. Normal saline ( $10 \mathrm{ml} / \mathrm{kg}$, i.p.) treated rats of control group demonstrated significant decrease in ELT as compared to ELT on first day during acquisition trials conducted from day 1 to day 4 and significant increase TSTQ in search of missing platform as compared to other quadrants during retrieval trial on day 5 (Figure 3) (Figure 4). In contrast, chronic pre-treatment with HFEBOI (40 and $80 \mathrm{mg} / \mathrm{kg}$, p. o.) 15minutes before the administration of scopolamine hydrobromide produced significant decrease in the ELT to reach the platform in rats (Table 1) and increase in TSTQ in search of missing platform (Figure 4) as compared to scopolamine treated rats on respective day. The HFEBOI reversed significantly and dose dependently scopolamine induced memory deficit. Memory-enhancing activity of HFEBOI was comparable to piracetam. Scopolamine hydrobromide $(0.3 \mathrm{mg} / \mathrm{kg}$, i.p.) resulted in significant memory deficit in EPM and MWM tasks. It has also been used in preclinical stages of drug development and clinically as an adjunct to surgical or obstetric procedure to induce sedation and post-procedural amnesia. ${ }^{27}$ Scopolamine $(0.3 \mathrm{mg} / \mathrm{kg}$, i.p. $)$ is a central anti-cholinergic drug ${ }^{28}$ that blocks muscarinic cholinergic receptors, impairs acquisition and retrieval of memory as reflected by significantly decrease in TLT as IR in EPM, increase in ELT on four consecutive acquisition days and decrease in TSTQ on day 5 in MWM respectively as compared to control group. A significant decrease in the TLT of control group rats noted on the second day as compared to their TLT on the first day indicated normal memory in EPM. Similarly, a marked decrease in ELT of rats in control group, during subsequent acquisition trials as compared to first exposure on MWM, denotes normal acquisition of memory whereas an enhancement in TSTQ by the rat in search of the missing platform during retrieval trial indicates successful retrieval of memory. The administration of normal saline did not produce any modification in the EPM and MWM performance of control rats. Since, both of these different memory models produced uniform results on memory scores, the built-in limitation if any, present in an individual experiment model was automatically taken care of.

Table I Effect of HFEBOI on Scopolamine Induced Changes in ELT during acquisition trials

The ELT of hydro alcoholic extract of Brassica oleracea L. var. italic flower $(20 \mathrm{mg} / \mathrm{kg}$ and $40 \mathrm{mg} / \mathrm{kg})$, conducted on four consecutive days are shown. It is noted that the scopolamine has a significantly increasing effect on ELT. Hydro alcoholic extract of the flower and piracetam have a decreasing effect. Each value of ELT is a mean value of four consecutive acquisition trials conducted day I to day 4. Each group $(n=6)$ represents mean $\pm S$. E.M. $a=p \leq 0.05$ Vs ELT on day I, $b=p \leq 0.05 \quad E L T$ in control group on respective day and $c=p \leq 0.05 \mathrm{Vs}$ ELT in scopolamine treated group. One way ANOVA test was employed.

\begin{tabular}{|c|c|c|c|c|c|c|}
\hline \multirow{2}{*}{ Groups } & \multirow{2}{*}{ Treatment } & \multirow{2}{*}{$\operatorname{Dose}\left(\mathrm{Kg}^{-1}\right)$} & \multicolumn{4}{|c|}{ E LT (Sec) on acquisition days } \\
\hline & & & Day I & Day 2 & Day3 & Day 4 \\
\hline 1 & Control (Normal saline) & $10 \mathrm{ml}$ & $110.33 \pm 4.1$ & $106.5 \pm 5.8^{\text {a }}$ & $97.66 \pm 8.91^{\text {a }}$ & $84 . .50 \pm 5.33^{\text {a }}$ \\
\hline II & Scopolamine & $0.3 \mathrm{mg}$ & $120 \pm 4.60^{b}$ & $114 . \pm 6.09^{b}$ & $105 \pm 3.80^{b}$ & $95 \pm 3.24^{b}$ \\
\hline III & Piracetam & $400 \mathrm{mg}$ & $108 \pm 5.6$ & $104.75 \pm 2.1$ & $94.5 \pm 2.53$ & $80.75 \pm 2.46$ \\
\hline IV & HFEBOI & $40 \mathrm{mg}$ & $103.52 \pm 6.44$ & $98.25 \pm 6.57^{a, c}$ & $78 \pm 2.62^{a, c}$ & $50.75 \pm 3.25^{\mathrm{a}, \mathrm{c}}$ \\
\hline v & HFEBOI & $80 \mathrm{mg}$ & $90 \pm 4.56^{\mathrm{a}, \mathrm{c}}$ & $89.75 \pm 4.74 \mathrm{a}, \mathrm{c}$ & $87.75 \pm 1.96^{\mathrm{a}, \mathrm{c}}$ & $74.75 \pm 3.4^{\mathrm{a}, \mathrm{c}}$ \\
\hline VI & Piracetam+Scopolamine & $400+0.3 \mathrm{mg}$ & $75.25 \pm 5.5^{c}$ & $62.12 \pm 6.25^{c}$ & $56.25 \pm 4.5^{c}$ & $50 \pm 10.75^{c}$ \\
\hline VII & HFEBOI+Scopolamine & $40+0.3 \mathrm{mg}$ & $110.25 \pm 6.0$ & $80.25 \pm 3.22^{c}$ & $79.5 \pm 2.28^{c}$ & $72.5 \pm 2.33^{c}$ \\
\hline VIII & HFEBOI+Scopolamine & $80+0.3 \mathrm{mg}$ & $107 \pm 1.58^{c}$ & $103 \pm 1.29 c$ & $72.75 \pm 1.10^{c}$ & $63.25 \pm 1.37^{c}$ \\
\hline
\end{tabular}




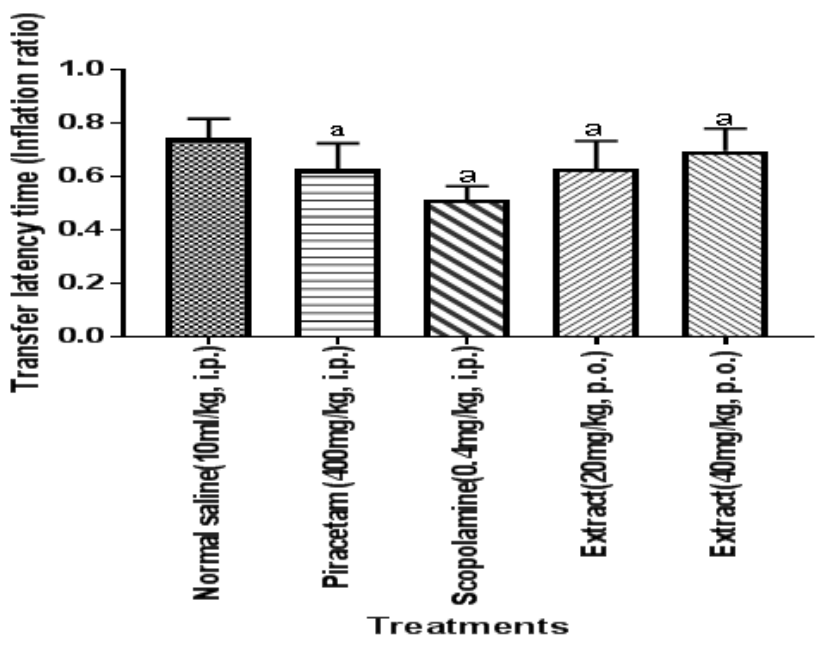

Figure I Effect of HFEBOI per se on scopolamine induced change in TLT as IR.

The effect of hydro alcoholic extract of Brassica oleracea L. var. italic flower $(40 \mathrm{mg} / \mathrm{kg}$ and $80 \mathrm{mg} / \mathrm{kg})$ on TLT as IR was observed in young male rats using EPM. TLT as IR conducted on after 24hrs of acquisition trial (on the $5^{\text {th }}$ day) indicates memory retention. It is noted that the scopolamine has a significant decreasing effect on memory retention. HFBOI and piracetam have an increasing effect on memory retention. Each group $(n=6)$ represents mean \pm S.E.M. $a=p \leq 0.05 \mathrm{Vs}$ TLT as IR in control group. One way ANOVA test was employed.

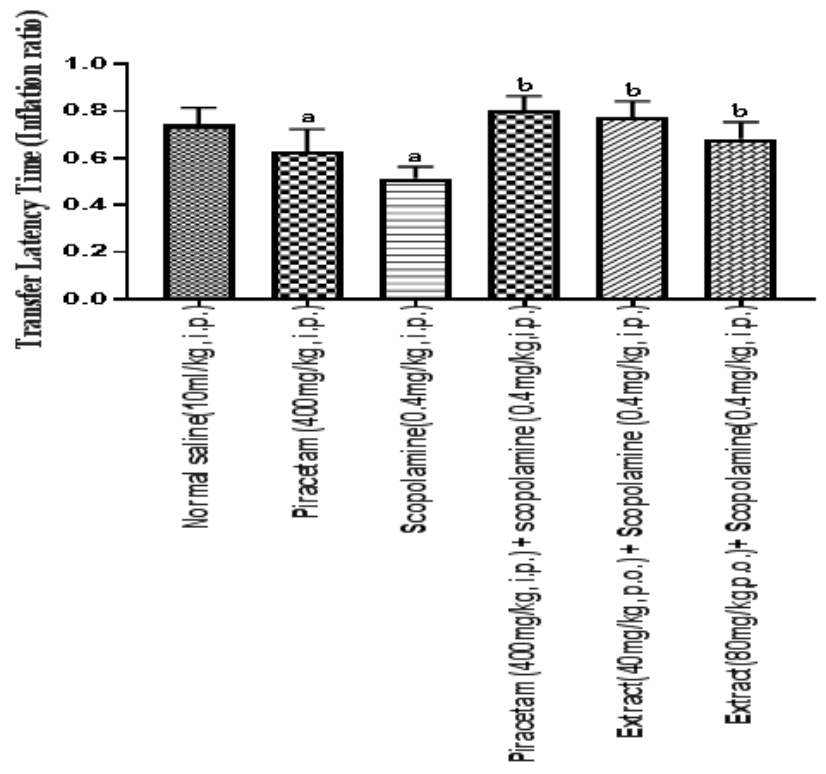

\section{Treatments}

Figure 2 Effect of HFEBOI on TLT as IR in scopolamine induced memory deficit.

The effect of hydro alcoholic extract of Brassica oleracea $L$. var. italic flower $(40 \mathrm{mg} / \mathrm{kg}$ and $80 \mathrm{mg} / \mathrm{kg})$ on TLT as IR was observed against scopolamine induced memory deficit rats using EPM. TLT as IR conducted on after $24 \mathrm{hrs}$ of acquisition trial (on the $5^{\text {th }}$ day) indicates memory retention. It is noted that the scopolamine has a significant decreasing effect on memory retention. HFBOI and piracetam have an increasing effect on memory retention. Each group $(n=6)$ represents mean \pm S.E.M. $a=p \leq 0.05$ versus $T L T$ in control group $b=p \leq 0.0 \mathrm{Vs}$ TLT in scopolamine treated group. One way ANOVA test was employed.

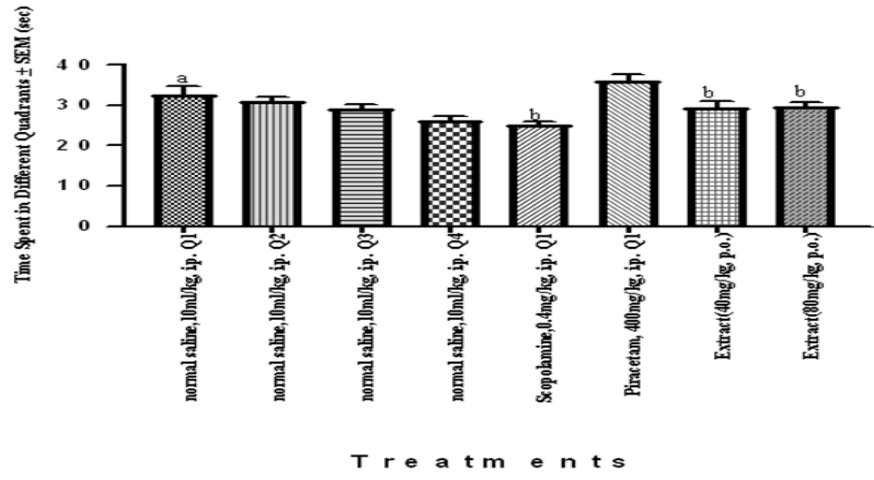

Figure 3 Effect of HFEBOI on TSTQ during retrieval trial using MWM.

Piracetam $(400 \mathrm{mg} / \mathrm{kg}$, i.p.), Brassica oleracea L. $(40 \mathrm{mg} / \mathrm{kg}$, p. o. and $80 \mathrm{mg} / \mathrm{kg}$, p. o.) groups increased time spent in target quadrant and showed learning. Scopolamine treated young rat decreased time spent in target quadrant as compared to QI target quadrant of control group. Results were expressed as mean \pm S.E.M with $\mathrm{n}=6$ in each group. $\mathrm{a}=\mathrm{p} \leq 0.05$ versus time spent in other quadrants, $b=p \leq 0.05$ versus time spent in target quadrant (QI) of control group. One way ANOVA test was employed.

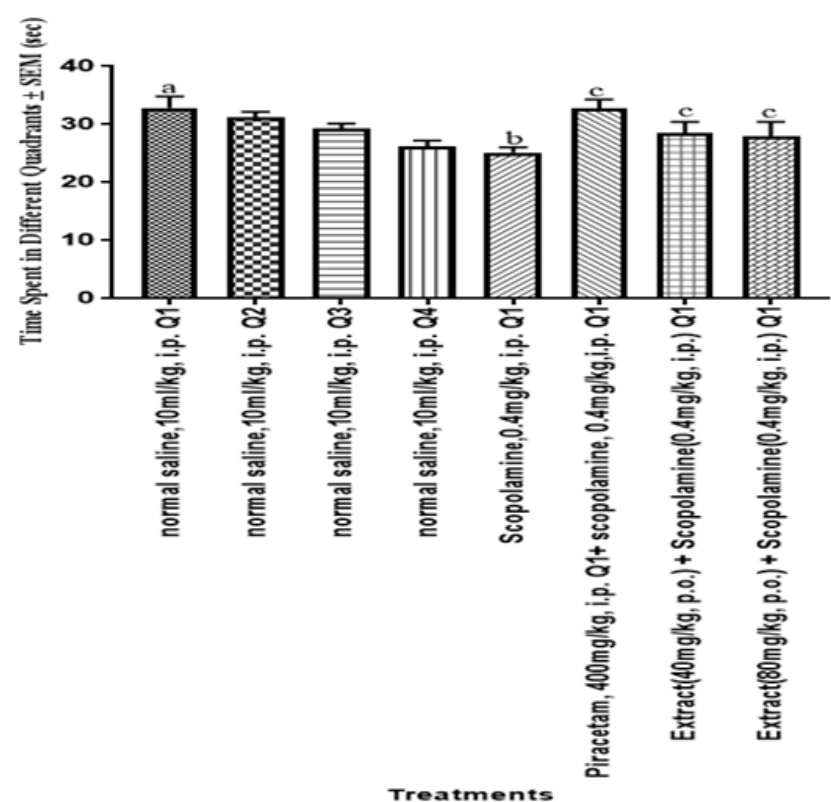

Figure 4 Effect of HFEBOI on scopolamine induced decrease in TSTQ during retrieval trial using MWM.

Piracetam $(400 \mathrm{mg} / \mathrm{kg}$, i.p.) + scopolamine $(0.3 \mathrm{mg} / \mathrm{kg}$, i.p.), HFBOI $(40 \mathrm{mg} / \mathrm{kg}$, p.o. and $80 \mathrm{mg} / \mathrm{kg}$, p.o.)+scopolamine treated group increased TSTQ and showed protection against scopolamine induced memory deficit. Scopolamine treated young rats decreased TSTQ as compared to target quadrant of control group. Results were expressed as mean \pm S.E.M with $n=6$ in each group. $a=p \leq 0.05$ versus time spent in other quadrants, $b=p \leq 0.05$ versus time spent in target quadrant in control group. $c=p \leq 0.05$ versus time spent in scopolamine treated group. One way ANOVA test was employed.

\section{Antioxidant Effect of Brassica oleracea L. var. Italica in scopolamine treated rats}

First, a decrease in acetylcholine (ACh), a cholinergic neurotransmitter essential for the process of normal learning, memory and attention as well as a decrease in level of natural antioxidants in the brain by activating microglia, a source of reactive oxygen species (ROS), has been reported. ${ }^{29}$ The formation of ROS and other free 
radicals during metabolism is an important and normal process that is ideally compensated by an elaborate endogenous antioxidant system. However, excessive radical production and their accumulation result in oxidative stress, which has been implicated in mechanisms responsible for oxidative injury of neurons by causing damage of cell structures, including lipids, membranes, and proteins. ${ }^{30}$ The central nervous system is very susceptible to oxidative stress. Additionally, it contains large amounts of free-radical generating substances like iron, ascorbate, glutamate and unsaturated fatty acids that easily undergo redox-reaction leading to radical formation. ${ }^{31}$ An increase in free radicals causes overproduction of MDA. MDA, the final product of the polyunsaturated fatty acids per-oxidation in the cells, is a neuronal toxin and may impair protein function. ${ }^{32}$ Lipids per-oxidation refers to the oxidative degradation of lipids. It is the process whereby free radicals 'steal' electrons from lipids from the cell membranes resulting in cell damage. Per-oxidation of lipids, which are abundant constituent of neurilemma, can directly destroy the structural integrity of membranes and lead to significant changes in their biophysical functions. It has been reported that the level of MDA, marker of oxidative stress is generally higher in senile dementia. Certain diagnostic tests specifically MDA are available for the quantification of end products of lipids per-oxidation. Glutathione is an endogenous antioxidant present in the reduced form within the cells. It protects cells from reactive oxygen species such as hydroxyl free radicals and peroxidase. ${ }^{33}$ The decreased level of GSH in scopolamine-treated animals indicates that there is an increased generation of free radicals and reduced activity of glutathione system in combating oxidative stress. Accumulation of nitrite is an indicator of nitric oxide, which is produced due to oxidative stress in the brain. Nitric oxide also acts as a precursor for peroxynitrite free radical which results in neuronal damage combating oxidative stress. Free radicals are the most likely candidates responsible for producing neuronal changes mediating the behavioral deficits in Alzheimer's disease. ${ }^{34,35}$ Intense oxidative stress related processes (imbalances between local ROS and antioxidant capacity, neuroinflammation, and accumulation of oxidatively modified proteins) within the brain potentiate neurodegeneration and impair cognitive function causing memory deficit. ${ }^{36}$ Biomarkers are a significant tool to identify high-risk individuals, to diagnose disease conditions promptly and accurately, and to prognosticate efficiently and treat patients with disease. Hence, MDA, nitrite and glutathione biomarkers estimations were carried out in order to verify antioxidant status of the HFBOL. Scopolamine caused significant rise in brain MDA, nitrite levels and depletion of GSH levels as compared to normal saline. Chronic HFEBOI (40 and $80 \mathrm{mg} / \mathrm{kg}$, p.o.) treatment significantly attenuated the increase in MDA (Figure 5), nitrite levels (Figure 6) and restored decrease in GSH (Figure 7). Broccoli has been reported to be good source of polyphenolic [4-(1-methylpropyl) phenol, 4-methylphenol, 2-methoxy-4-vinylphenol \& ferulic phenol acid], vitamins [A,C \& E]; and flavonoids [quercetin-3- $O$-sophorotrioside7-O-glucoside, beta-carotene cryptoxanthin, lutein, and zea-xanthin] antioxidants in diet which are major contributors in particular to its anti-oxidative activity. ${ }^{37}$ Broccoli sprout extract contained $1 \%$ suloraphane decreased urinary 8-hydroxy-2-deoxyguanosine ${ }^{38}$ and hexanoyl-lysine adduct ${ }^{39}$ and is effective to inhibit oxidative DNA damage and initial stage of lipid peroxidation in-vivo respectively in treatment of ischemic stroke. The chloroform fraction of broccoli leaf extract had the highest total phenolic content and the highest 2, 2"-azino-bis (3-ethylbenzothiazoline-6-sulfonic acid), diammonium salt radical-scavenging activity and malondialdehyde (MDA) inhibitory effect. ${ }^{40}$ In-vitro models clearly suggest that, broccoli is a natural source for antioxidants, which could serve as a nutraceutical with potential applications in reducing the level of oxidative stress and related health benefits.

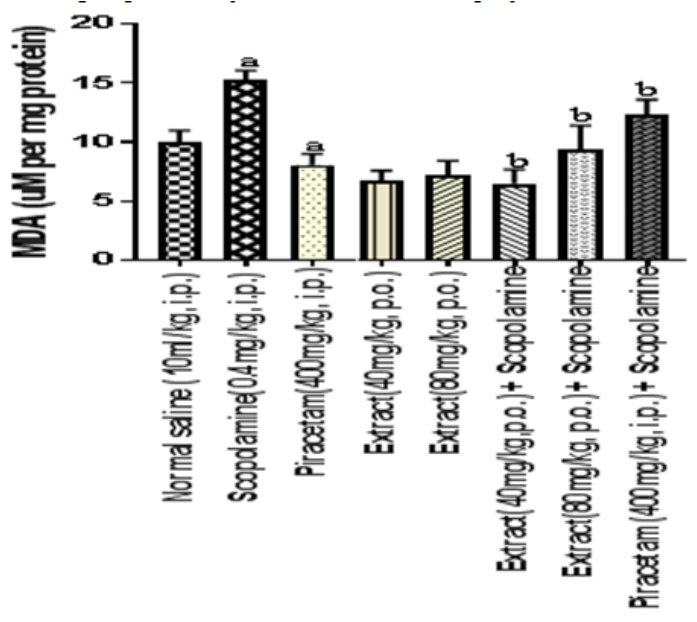

Figure 5 Effect of HFEBOI on brain MDA level.

Bar graph represents the effect of HFBOI on MDA level: as compared to control group, significant increase in MDA level was noted in scopolamine treated group. Piracetam, HFBOI $(40 \mathrm{mg} / \mathrm{kg}$, p.o. and $80 \mathrm{mg} / \mathrm{kg}$, p.o.) treated group decreased brain MDA level. Results were expressed as mean with $n=6$ each group. $a=p \leq 0.05$ versus brain MDA level in normal saline treated control group, $b=p \leq 0.05$ versus brain MDA level in sopolamine treated group.

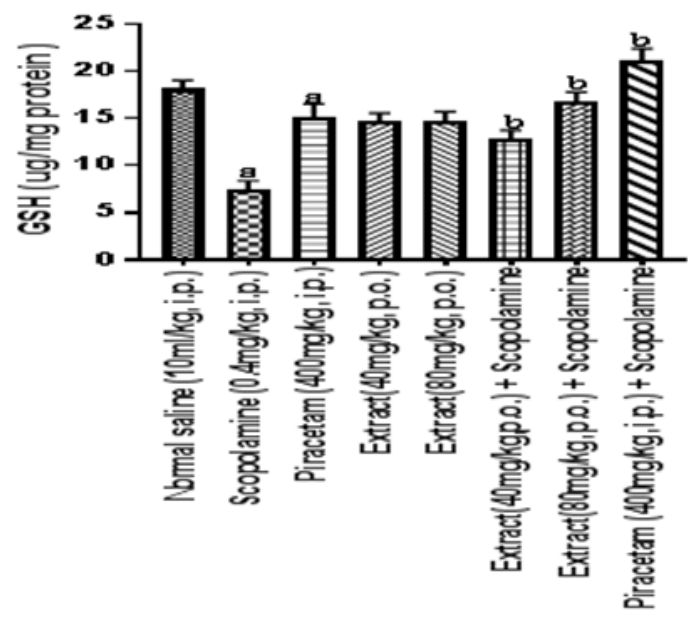

Figure 6 Effect of HFEBOI on brain glutathione level.

Bar graph represents the effect of HFBOI on glutathione level, as compared to control group in scopolamine treated group significant decrease in GSH level was noted. Piracetam, HFBOI $(40 \mathrm{mg} / \mathrm{kg}$, p.o. and $80 \mathrm{mg} / \mathrm{kg}$, p.o.) treated groups restored brain GSH level. Results were expressed as mean with $n=6$ each group. $a=p \leq 0.05$ versus brain GSH level in normal saline treated control group, $b=p \leq 0.05$ versus brain GSH level in scopolamine treated group.

\section{Reversal of increased brain AChE by Brassica oleracea L. var. Italica in scopolamine treated rats}

Normal saline did not show any significant effect on brain AChE level in control group. In contrast scopolamine, a central anti-cholinergic drug showed significant increase in the brain AChE level as compared to normal saline treated rats. However, chronic oral administration of Brassica oleracea L. var. Italica $(40 \mathrm{mg} / \mathrm{kg}$ and $80 \mathrm{mg} / \mathrm{kg}$, po) significantly attenuated enhanced AChE level as compared to scopolamine treated group (Figure 8). Salient findings of present study are that the enhanced $\mathrm{AChE}$ activity was normalized by HFEBOI pre-treatment, thereby increasing ACh content in brain, which was reported necessary to reduce cognitive dysfunction. 
Broccoli contains diindolylmethane, which possessed anti-ChE activity. Drugs that inhibit AChE enzyme, protects acetylcholine from hydrolysis and may increase the level of acetylcholine in the brain and have nootropic activity. ${ }^{41,42}$ Hence, anti-dementic activity of the flower extract may be mediated through facilitation of cholinergic transmission by its diindolylmethane and antioxidant effect.

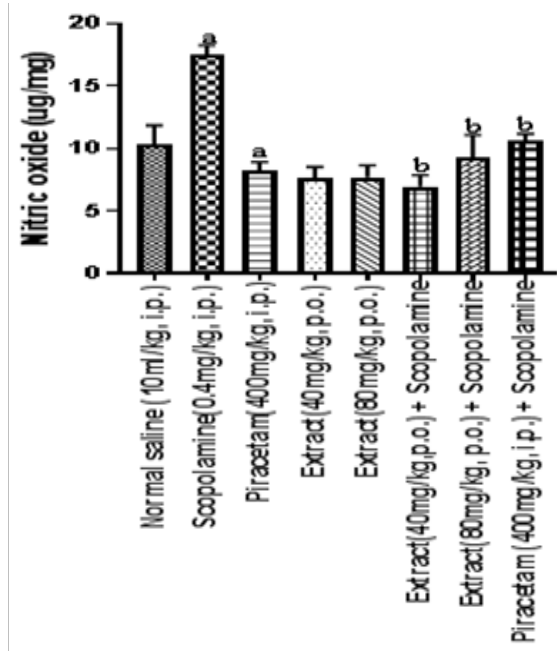

Figure 7 Effect of HFEBOI on brain nitrite level.

Bar graph represents the effect of HFBOI on nitrite level, As compared to control group, significant increase in nitrite level was noted in scopolamine treated group. Piracetam, HFBOI $(40 \mathrm{mg} / \mathrm{kg}$, p.o. and $80 \mathrm{mg} / \mathrm{kg}$, p.o.) treated group decreased nitrite level. Results were expressed as mean \pm S.E.M. with $n=6$ in each group. $a=p \leq 0.05 \mathrm{Vs}$ NO level in normal saline treated control group; $b=p \leq 0.05 \mathrm{Vs}$ NO level in scopolamine treated group.

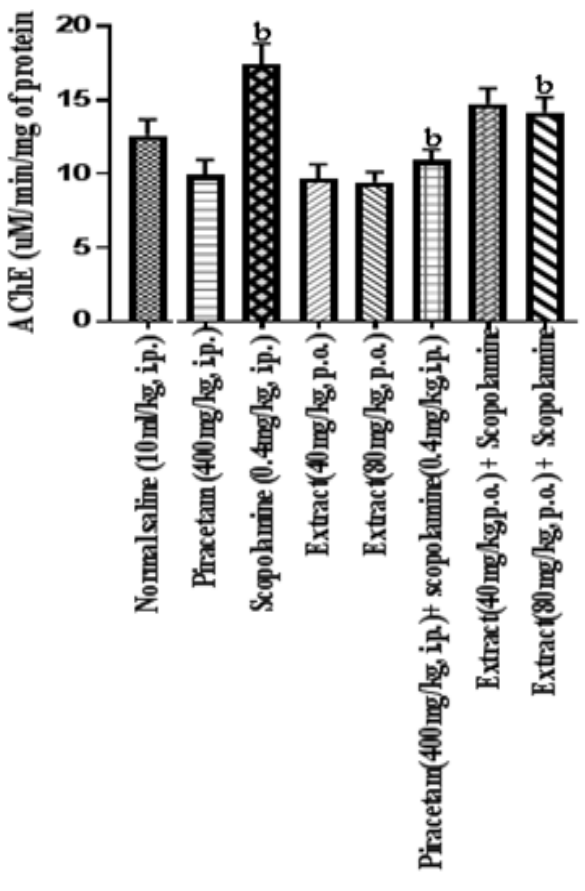

Figure 8 Effect of HFEBOI on brain AChE level.

Bar graph represents the effect of HFBOI on brain AChE level. As compared to control group, significant increase in $\mathrm{AChE}$ level was noted in scopolamine treated group. Piracetam, HFBOI (40 and $80 \mathrm{mg} / \mathrm{kg}$, p.o.) treated group normalized $A C h E$ level. Results were expressed as mean with $n=6$ each group. $a=p \leq 0.05$ versus brain $A C h E$ activity of control group, $b=p \leq 0.05$ versus brain AChE activity of control group.

\section{Conclusion}

In summary, the present study suggests that chronic administration of the flower extract at both doses exhibited improvement in normal working memory in EPM and spatial memory respectively; prevented scopolamine induced memory deficit $\&$ associated oxidative stress. Increase in AChE, MDA and nitrate levels was attenuated but decrease in the glutathione level was reversed by the flower extract as compared to scopolamine treated group. It can be concluded that chronic HFEBOI pretreatment exhibited improvement in both acquisition and retrieval components of scopolamine induced memory deficit in young male rats by its potent anti-ChE and antioxidant activity. There is no experiment that actually demonstrates that anti-ChE and antioxidant activity are the mechanisms for the antidementic activity of Brassica oleracea L. var. italic. In previous study, chloroform fraction of broccoli leaf extract has been reported to ameliorate $\mathrm{A} \beta_{1 \text {. }}$ ${ }_{42}$ induced learning and memory impairment, to enhance cognitive function evaluated by Y-maze, passive avoidance, and MWM tests. and inhibition against $\mathrm{AChE} .{ }^{43}$ It may be speculated that consumption of the broccoli may have potential for the management of dementia clinically. The limitations of study are summarized as follows. Since oestrogen, a female sex hormone has been found to have effect on memory; female rats were excluded and only male rats were used for the study. Scopolamine does not discriminate very much with respect to brain region. EPM and MWM Models require intact working and reference memory. Motivational stimuli $(50 \mathrm{~cm}$ elevation of maze from floor in EPM and water in MWM) exist outside the body of the animal. Water provides uniform environment and eliminates interference due to olfactory clues. The experimenter always stood at the same position. Care was taken that relative location of water maze with respect to other objects in the laboratory, serving as prominent visual clues was not disturbed during the total duration of study.

\section{Acknowledgements}

None.

\section{Conflict of interest}

Author declares that there is no conflict of interest.

\section{References}

1. Harrison N, Lange L, Barker JL. Pre and post synaptic aspects of GABAmediated syneptic inhibition in cultured cell hippocampal neurons. $A d v$ Biochem Psychopharmacol. 1998;45:73-85.

2. Lanzino G, Lanzino DJ, Wang D. Cerebrovascular disease and cognitive dysfunction. Neurol Res. 2002;24:331-336.

3. Tripathi KD. Essentials of Medical Pharmacology. 7th ed. New Delhi: J. P. Brothers Medical Publishers (P) LTD; 2013. p. 486-491.

4. Bigio EH, Hynan LS, Sontag E, et al. Synapse loss is greater in presenile than senile onset Alzheimer disease: implications for the cognitive reserve hypothesis. Neuropathol Appl Neurobiol. 2002;28(3):218-227.

5. Howlett DR, Simmons L, Dingwall C, et al. In search of an enzyme: the $\beta$-secretase of Alzheimer's disease is an aspartic proteinase. Trends Neur. 2000;23(11):565-570.

6. Jotheeswaran AT, Williams JD, Prince MJ. The predictive validity of the 10/66 dementia diagnosis in Chennai, India: a 3-year followup study of cases identified at baseline. Alzheimer Dis Assoc Disord. 2010;24(3):296-302.

7. Weinstock M. Selectivity of cholinesterase inhibition: Clinical implication for the treatment of Alzheimer's disease. J Neurol Neurosurg Psychiatry. 1999;12(1):303-307. 
8. Yoshida S, Suzuki N. Antiamnesic and cholinomimetic side-effects of the cholinesterase inhibitors, physostigmine, tacrine and NIK-247 in rats. Eur J Pharmacol. 1993;250(1):117-124.

9. Scartezzini P, Speroni E. Review on some plants of Indian traditional medicine with antioxidant activity. J Ethnopharmacol. 2000;71(1-2):23-43.

10. Singh N, Pandey BR, Verma P. An overview of phytotherapeutic approach in prevention and treatment of Alzheimer's Syndrome and Dementia. Int J Pharma Sci Drug Res. 2011;3(3):162-172.

11. Mukherjee V, Mishra PK. Broccoli-an underexploited. Neutraceutical Sci Res Reporter. 2012;2(3):291-294.

12. Lim TK. Edibile medicinal and non-medicinal plants Volume 7 Flowers. New York: Springer Dordrecht Heidelberg; 2014:616.

13. Sibi G, Shukla A, Dhananjaya K, et al. In-vitro antibacterial activities of Broccoli (Brassica oleracea L.var italica) against food borne bacteria. JAPS. 2013;3(5):100-103.

14. Kusuma MA, Sakinah EN, Dewi R. Hepatoprotective effect of ethanolic extract of Broccoli (Brassica oleracea L. var. italic) on histologically damage of wistar rats liver cell induced by DMBA. JPK. 2017;5(1):6-11.

15. Owis AI. Broccoli; the green beauty: a review. J Pharm Sci \& Res. 2015;7(9):696-703.

16. Gaby AR. Natural approaches to epilepsy. Altern Med Rev. 2007;12(1):9-24.

17. Krebs-Smith SW. Choose a variety of flowers and vegetables daily: understanding the complexities. |J Nutr. 2001;31(1):487s-501s.

18. Ong ES. Extraction methods and chemical standardization of botanicals and herbal preparations. J Chromatogr B. 2004;812(1-2):23-33.

19. Lala PK. Lab Manuals of Pharmacognosy. 5th ed. Calcutta: CSI Publishers and Distributors; 1993.

20. Safer DJ, Allen RP. The central effects of scopolamine in man. Biol Psychiatry. 1971;3(4):347-55.

21. Goverdhan P, Sravanthi A, Mamatha T. Neuroprotective effects of meloxicam and selegiline in scopolamine-induced cognitive impairment and oxidative stress. Int $J$ Alzheimers Dis. 2012;2012:1-8.

22. Morris R. Developments of a water maze procedure for studying spatial learning in the rat. $J$ Neurosci Methods. 1984;11(1):47-60.

23. Okhawa H, Ohishi N, Yagi K. Assay for lipid per-oxidation in animal tissue by thiobarbituric acid reaction. Anal Biochem. 1979;95(2):351-358.

24. Green LC, Green DA, Wagner J, et al. Analysis of nitrate nitrite and nitrate in biological fluids. Anal Biochem. 1982;126(1):131-138.

25. Anderson ME. Determination of glutathione and glutathione disulfide in biological samples. Methods Enzymol. 1985;113:548-555.

26. Ellman GL, Courtney Kd, Andres V Jr, et al. A new and rapid colorimetric determination of acetylcholinesterase activity. Biochem Pharmacol. 1961;7:88-95.

27. Safer DJ, Allen RP. The central effects of scopolamine in man. Biol Psychiatry. 1971;3(4):347-355.
28. Kulkarni SK, Kasture SB, Mengi SA. Efficacy study of Prunus amygdalus (almond) nuts in scopolamine-induced amnesia in rats. Indian J Pharmacol. 2010;42(3):168-173.

29. $\mathrm{Lu} \mathrm{SH}, \mathrm{Wu}$ JW, Liu HL, et al. The discovery of potential acetylcholinesterase inhibitors: a combination of pharmacophore modeling, virtual screening, and molecular docking studies. $J$ Biomed Sci. 2011;18(1):1-13.

30. Calabrese V, Butterfield DA, Stella AM. Nutritional antioxidants and the heme oxygenase pathway of stress tolerance: novel targets for neuroprotection in Alzheimer's disease. Ital J Biochem. 2003;52(4):177-181.

31. Walton NM, Shin R, Tajinda K, et al. Adult neurogenesis transiently generates oxidative stress. PLoS ONE. 2012;7(4):e35264.

32. Agostinho P, Cunha RA, Oliveira C. Neuroinflammation, oxidative stress and the pathogenesis of Alzheimer's disease. Curr Pharm Des. 2010;16(25):2766-2778

33. Bains JS, Shaw CA. Neurodegenerative disorders in humans: the role of glutathione in oxidative stress-mediated neuronal death. Brain Res Rev. 1997;25(3):335-358.

34. Cantuti-Castelvetri I, Shukitt-Hale B, Joseph JA. Neurobehavioral aspects of antioxidants in aging. Int J Dev Neurosci. 2000;18(4-5):367-381.

35. Maxwell SR. Prospects for the use of antioxidant therapies, Drugs. 1995;49(3):345-361.

36. Kruk-Slomka M, Boguszewska-Czubara A, Slomka T, et al. Correlations between the memory-related behaviour and the level of oxidative stress biomarkers in the mice brain, provoked by an acute administration of $\mathrm{CB}$ receptor ligands. Neural Plast. 2016;2016:1-15.

37. Upadhyay R, Sehwag S, Singh SP. Antioxidant activity and polyphenol content of Brassica oleracea varieties, Int J Vegetables Sci. 2015;22(4):353-363.

38. Nakajima H, Unoda K, Ito T, et al. The relation of urinary 8-OHD, a marker of oxidative stress to DNA and clinical outcomes for ischemic stroke. Open Neurol J. 2012;6:51-57.

39. Jones DP. Redefining oxidative stress. Antioxid Redox Signal. 2006;8(9-10):1865-1879.

40. Park SK, Jin DE, Park CH, et al. PC12 cell protective effects of broccoli (Brassica oleracea var. italica) leaf fraction against $\mathrm{H}_{2} \mathrm{O}_{2}$-induced oxidative stress Korean. J Food Sci Technol. 2014;46(4):483-488.

41. Kopelman MD. Disorders of memory. Brain. 2002;125(10):2152-2190.

42. Kumar A, Dogra S, Prakash A. Neuroprotective effects of Centella asiatica against intracerebroventricular colchicine-induced cognitive impairment and oxidative stress. Int J Alzheimers Dis 2009:1-8.

43. Park SK, Ha JS, Kim JM, et al. Antiamnesic Effect of broccoli (Brassica oleracea var. italica) leaves on amyloid beta $(\mathrm{A} \beta) 1$-42-induced learning and memory impairment. J Agric Food Chem. 2016;64(17):3353-3361. 